\title{
An overview of ultrasonography in lateral epicondylitis: Correlation with disease duration and severity
}

\author{
Fatma Nur Soylu Boy ${ }^{1}$, Feyza Unlu Ozkan ${ }^{2}$, Duygu Geler Kulcu ${ }^{3}$, Hakki Muammer Karakas ${ }^{1}$, \\ Muhittin Mumtaz Ozarar ${ }^{1}$, Bulent Kilic ${ }^{4}$, I lknur Aktas ${ }^{2}$
}

1. Fatih Sultan Mehmet Training and Research Hospital, Department of Radiology, Istanbul, Turkey. 2. Fatih Sultan Mehmet Training and Research Hospital, Department of Physical Medicine and Rehabilitation, Istanbul, Turkey. 3. Haydarpasa Numune Training and Research Hospital, Department of Physical Therapy and Rehabilitation, Istanbul, Turkey. 4. Istanbul Gelisim University, Department of Health Sciences, Istanbul, Turkey

Correspondence: Fatma Nur Soylu Boy. Address: Fatih Sultan Mehmet Egitim ve Arastirma Hastanesi, E-5 Karayolu Uzeri, Istanbul, Turkey. Email: nursoylu@yahoo.com

Received: June 24, 2015

DOI : $10.5430 /$ ijdi.v3n1p1
Accepted: August 15, 2015

URL: http://dx.doi.org/10.5430/ijdi.v3n1p1

Online Published: August 20, 2015

\section{Abstract}

The purpose of this study is to make an overview for ultrasonographic features of lateral epicondylitis and to investigate the correlation of these features with clinical findings including disease duration and severity. This prospective study included 42 consecutive patients diagnosed with lateral epicondylitis on clinical examination. Three patients had bilateral involvement, therefore, 45 elbows were examined with ultrasonography. In addition, the asymptomatic contralateral 39 elbows of 39 patients and as a control group, 16 elbows of 16 healthy asymptomatic volunteers were examined. The ultrasonographic features of lateral epicondylitis in common extensor tendon including focal hypoechoic area, heterogenicity, focal areas of calcification, increase or decrease in thickness, partial or complete tear, peritendinous fluid collection and entesophyte were investigated. These findings were compared with the clinical findings including the duration and severity of complaint. Visual analog scale (VAS) was used to evaluate the severity of pain. Fisher exact test and Mann-Whitney U test were used in statistical analysis, and all tests were considered significant at $p<.05$ in $95 \%$ CIs. In the diseased elbows, the most common finding of the lateral epicondylitis was heterogenicity, focal hypoechoic area and entesophyte, respectively. Statistical examination revealed no significant correlation between ultrasonographic findings and duration of the symtoms. There was also no significant correlation between ultrasonographic findings and severity of pain. Ultrasonography can demonstrate well the features of lateral epicondylitis. It could also be helpful to show the extent of the disease. However, ultrasonographic findings do not significantly correlate with duration and severity of the disease clinically.

\section{Keywords}

Lateral epicondylitis, Elbow, Ultrasonography

\section{I ntroduction}

Lateral epicondylitis is the most common cause of lateral elbow pain which typically occurs in the 4th and 5th decades of life $^{[1-3]}$. Lateral epicondylitis occurs due to the chronic stress of common extensor tendon which originates at the lateral Published by Sciedu Press 
epicondyle. Although lateral epicondylitis is also known as the tennis elbow, in great majority of cases it is seen in non-tennis players ${ }^{[3]}$.

Daily use of ultrasonography has been increased recently in musculoskeletal diseases because it is a noninvasive and inexpensive technique providing high resolution and dynamic imaging, its use in lateral epicondylitis is still limited, since the diagnosis of lateral epicondylitis is easily made by clinical and physical examination. However, ultrasonography accurately demonstrates the morphology and echogenicity of common extensor tendon and its origin. Ultrasonography also allows the assessment of severity and location of the injury ${ }^{[1,3]}$ and can be used to confirm the diagnosis in cases with clinically equivocal cases ${ }^{[3]}$. We think that highlighting the correlation of extent of disease in ultrasonography with clinical symptoms is necessary, especially in patients who are candidate for surgery cause of high degree of pain or late stage disease. This study is designed to review the ultrasonographic features of lateral epicondylitis and to demonstrate ultrasonography as a feasible technique in the evaluation of lateral epicondylitis. In this manner, our study attempted to make the radiologists and clinicians more familiar with effective use of ultrasonography in this disease. Investigation of correlation between ultrasonographic features and clinical findings including duration and severity of the disease was also aimed which is especially would be important to avoid unnecessary operations.

\section{Materials and methods}

\subsection{Patient population}

This prospective study was approved by the institutional ethical board. Written informed consent was obtained from all participants. Between October 2013 and February 2014, 42 consecutive patients (mean age: 46.4 years, range: 18-64 years, 22 men and 20 women) diagnosed with lateral epicondylitis on clinical examination were evaluated. Three patients had bilateral involvement, hence 45 elbows were examined with ultrasonography. In 39 patients with unilateral disease the contralateral asymptomatic 39 elbows were also evaluated with ultrasonography. As a control group, 16 elbows in 16 healthy volunteers (mean age: 32.5 years, range: $22-62$ years, 9 men and 7 women) underwent ultrasonographic examination. These volunteers had no signs or symptoms of lateral epicondylitis.

\subsection{Clinical assessment}

Patients referring to the physical medicine and rehabilitation outpatient clinic with the symptoms of lateral epicondylitis were evaluated. Initial diagnosis was made on the basis of history and clinical findings. Physical examination included the presence of tenderness and pain over the lateral epicondyle. The duration of the symptoms were noted. Patients with complaints of 6 weeks or less were accepted as having early stage disease, and more than 6 weeks were accepted as having late stage lateral epicondylitis. Visual analog score (VAS) was used to evaluate the severity of pain during the day. The severity of the pain defined as "low" in patients with a VAS of $<5$ and "high" with a VAS of $\geq 5$.

Inclusion criteria for the study required the presence of lateral epicondylitis based on clinical examination and an age of 18-70 years. Exclusion criteria were history of elbow surgery, therapeutic steroid injection to the elbow, traumatic origin of lateral epicondylitis and collagen diseases.

\subsection{Ultrasonography technique and image interpretation}

All ultrasonography examinations were performed in high-resolution ultrasonography scanner (Acuson Antares, Siemens, Erlangen, Germany) with a multifrequency 5-13 MHz linear array transducer. All ultrasonography examinations were prospectively performed by the same radiologist (A, 9-year-experience with musculoskeletal ultrasonography). Ultrasonography examinations were performed while the subjects were lying in supine position with the examined elbow flexed to $135^{\circ}$. 
Common extensor tendon was examined in longitudinal and axial planes for demonstration of the internal architecture and echogenicity (see Figure 1). Ultrasonographic features of lateral epicondylitis in common extensor tendon including focal hypoechoic area, heterogen appereance, focal areas of calcification, the increase or decrease in the thickness, partial or complete tears, peritendinous fluid collections and entesophytes were investigated. A uniform fibrillar pattern followed from the muscle to the attachment side at lateral epicondyle was accepted as normal tendon echotexture. The focal decrease of normal echogenicity of the tendon, heterogen appereance defined as lack of normal fibrillar pattern or normal echotexture and calcifications were noted. The size of the tendon was assessed as either normal, enlarged or attenuated. A tendon was accepted as enlarged or attenuated if there was a $10 \%$ difference compared with the normal side in unilateral disease. A partial tear was defined as a focal fluid intensity oriented either horizontally or longitudinally in the common extensor tendon with the distortion of fibers. A complete fluid intensity extending through the full width of the common extensor origin was defined as a complete tear. Confirmation of an abnormality was performed in at least two planes during imaging. The examination time for an elbow was approximately 10 minutes. Asypmtomatic opposite elbow was also examined in patients with unilateral complaints. Ultrasonographic findings were compared with the clinical findings including the duration of complaints, and severity of pain.

Figure 1. Longitudinal Ultrasonography image of common extensor tendon in a 35-year-old healthy woman. The tendon has "uniform fibrillar pattern"” accepted as normal tendon echotexture and internal architecture

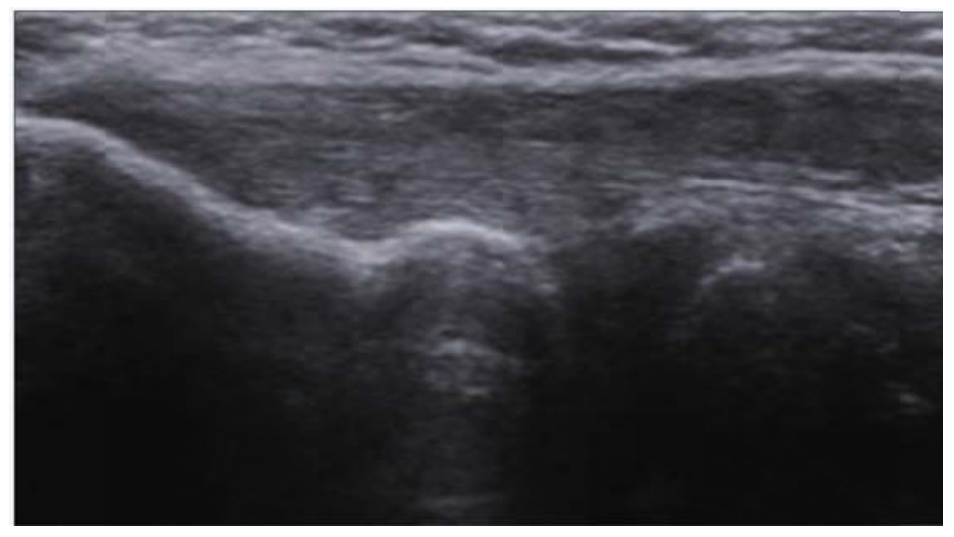

\subsection{Statistical analysis}

Statistical analysis was performed with NCSS 2007\&PASS 2008 software (Utah, USA).

Standard descriptive statistics were used to summarize characteristics of the patients and healthy volunteers. Ultrasonographic findings and severity of pain were compared by use of the Mann-Whitney U test. The comparison of ultrasonographic findings in patients with early and late stage disease was made by use of Fisher exact test. All tests were considered significant at $p<.05$ in $95 \%$ CIs.

\section{Results}

\subsection{Clinical findings}

In patients with lateral epicondylitis, 22 had right-sided lesions $(52.3 \%, 22 / 42)$ and 18 had left-sided lesions $(42.8 \%$, 18/42). Three patients had bilateral involvement. Twenty patients stated a repetetive injury from active use of the affected elbow including lifting, usign a device, typing, tennis and other sports. Twenty two patients did not give an obvious cause of injury. The interval from the initial symptoms to ultrasonography ranged from 4 weeks to 52 weeks (mean $16.8 \pm 13$ ). Sixteen elbows had early stage disease $(35.5 \%, 16 / 45)$ and 29 had late stage disease $(64.4 \%, 29 / 45)$. VAS had a range from 1 to 10 (mean $7.2 \pm 2.4$ ). Seventeen elbows were defined as having low degree of pain (VAS $<5 ; 37.7 \%, 17 / 45)$ and 28 
were defined as having high degree of pain (VAS $\geq 5,62.2 \%, 28 / 45)$. All patients had conservative treatment including rest, physical therapy, antiinflammatory drugs and steroid injections.

\subsection{Ultrasonography imaging findings}

Table 1 summarizes the ultrasonographic findings in patients diagnosed with lateral epicondylitis and contralateral asymptomatic elbows. Heterogenicity in common extensor tendon was the most common finding in diseased elbows followed by focal hypoechoic area (see Figure 2), entesophyte, focal areas of calcification (see Figure 3) and increase in thickness of the tendon, respectively. Partial tear (see Figure 4), complete tear and peritendinous fluid collection were less common findings. Besides, entesophyte was the most common finding in asymptomatic elbows, followed by heterogenicity, focal hypoechoic area, increased in the thickness and focal areas of calcification in the common extensor tendon. No patient had tendon tear or peritendinous fluid collection in asymptomatic elbows. Decrease in thickness of tendon was not observed in the patient group. In all control subjects, common extensor tendon had the normal echogenicity and internal architecture in ultrasonography.

Table 1. Ultrasonographic findings in patients diagnosed with lateral epicondylitis and contralateral asymptomatic elbows

\begin{tabular}{lll}
\hline & Elbows with Lateral epicondylitis (\%) & Asymptomatic Elbows (\%) \\
\hline Focal hypoechoic area & $35.5(16 / 45)$ & $17.9(7 / 39)$ \\
Heterogenicity & $40(18 / 45)$ & $20.5(8 / 39)$ \\
Focal areas of calcification & $13.3(6 / 45)$ & $2.5(1 / 39)$ \\
Partial tear & $6.6(3 / 45)$ & - \\
Complete tear & $2.2(1 / 45)$ & - \\
Peritendinous fluid collection & $2.2(1 / 45)$ & - \\
Increase in thickness & $13.3(6 / 45)$ & $7.6(3 / 39)$ \\
Entesophytes & $31.2(14 / 45)$ & $31(9 / 29)$ \\
\hline
\end{tabular}

Figure 2. Lateral epicondylitis in a 47-year-old man. Longitudinal US image showing a focal decrease in echogenicity adjacent to common extensor tendon origin defined as "focal hypoechoic area" (arrows)

Figure 3. Lateral epicondylitis in a 52-year-old woman. Longitudinal US image demonstrating a general decrease in echogenicity and lack of normal echotexture defined as "heterogenicity", foci of calcification within tendon (arrowheads) and entesophyte (arrow)
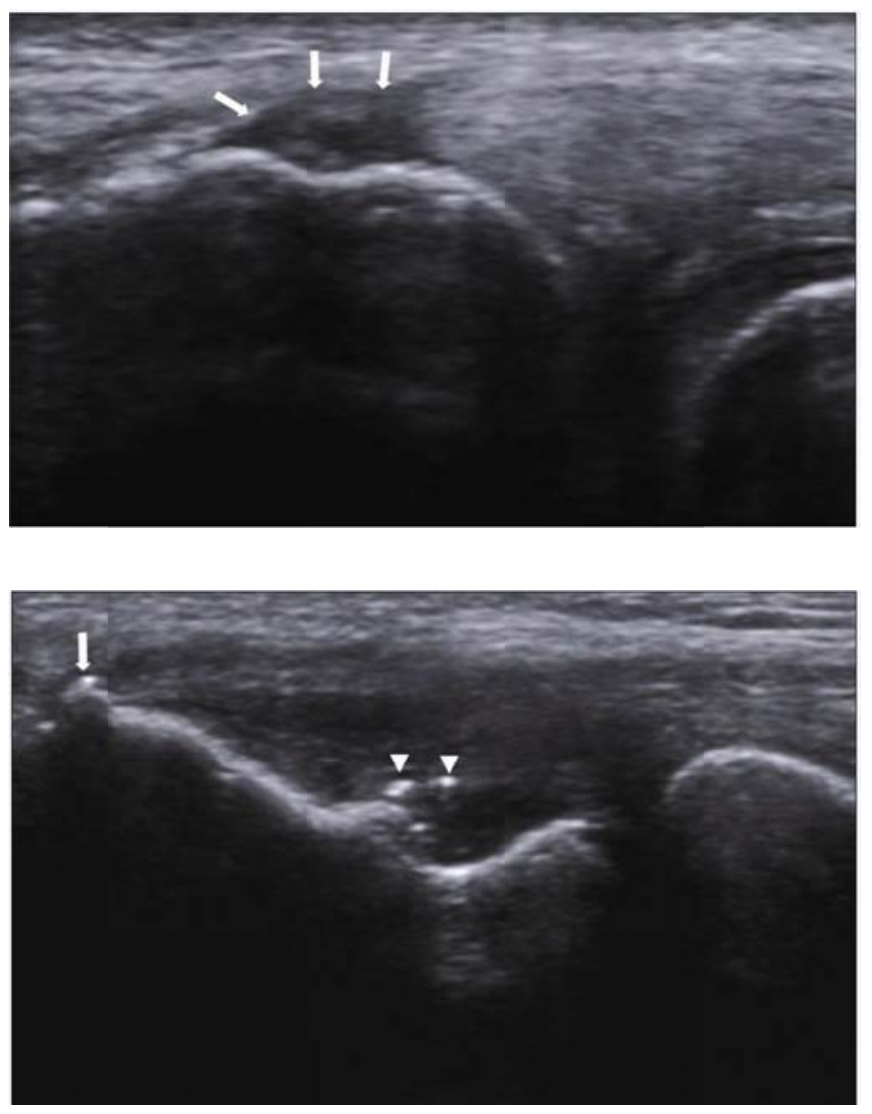

ISSN 2331-5857 E-ISSN 2331-5865 
Figure 4. Lateral epicondylitis in a 50-year-old woman. Longitudinal US image presenting general heterogenicity and focal fluid intensity in the common extensor tendon with the lack of fibers defined as "partial tear" (arrow)

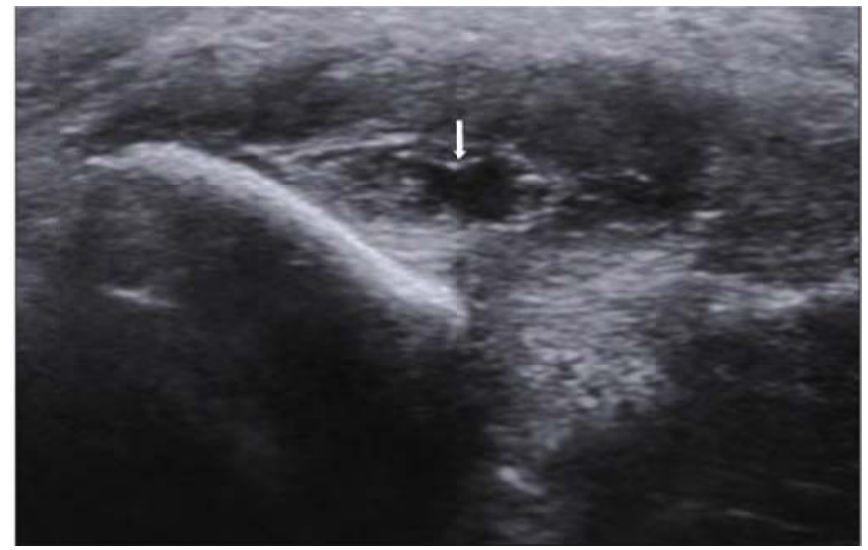

Table 2 summarizes the distribution of findings in patient group with early and late stages diseases. In early stage disease, focal hypoechoic area, heterogenicity and entesophytes were the most common findings in patients diagnosed with lateral epicondylitis. In late stage disease the heterogenicity was the most common finding followed by entesophytes. However, the statistical examination revealed no significant correlation between the ultrasonographic findings and the duration of the symtoms. There was also no significant correlation between ultrasonographic findings and severity of pain.

Table 2. The distribution of ultrasonographic findings in patients with early and late stages diseases

\begin{tabular}{llll}
\hline Ultrasonographic findings & Early Stage (\%) & Late Stage (\%) & $\boldsymbol{p}$ \\
\hline Focal hypoechoic area & $31.2(5 / 16)$ & $17.9(7 / 29)$ & .975 \\
Heterogenicity & $31.2(5 / 16)$ & $44.8(13 / 29)$ & .708 \\
Focal areas of calcification & $6.2(1 / 16)$ & $17.2(5 / 29)$ & .661 \\
Partial tear & $6.2(1 / 16)$ & $6.9(2 / 29)$ & .928 \\
Complete tear & - & $3.4(1 / 29)$ & - \\
Peritendinous fluid collection & - & $3.4(1 / 29)$ & - \\
Increase in tendon thickness & $6.2(1 / 16)$ & $17.2(5 / 29)$ & .426 \\
Entesophytes & $31.2(5 / 16)$ & $31(9 / 29)$ & .875 \\
\hline
\end{tabular}

Note. Numbers in parentheses were used to calculate percentages. The overall results were considered not statistically significant $(p \leq .05)$.

\section{Discussion}

Lateral epicondylitis is essentially a state of tendinosis which results from repetitive microtrauma and histologically corresponds to collagen degeneration, microtearing, reparation by granulation tissue and finally resulting partial or complete tears of the tendon ${ }^{[4-6]}$. The diagnosis of lateral epicondylitis is commonly based on clinical findings. However, ultrasonography of the common extensor tendon is usually used to confirm the diagnosis ${ }^{[1,3,4]}$. In ultrasonography, common extensor tendon is composed of the superificial extensor tendons of the forearm including extensor digitorum, extensor carpi ulnaris and extensor digiti minimi together with the extensor carpi radialis brevis and is characterized by uniform band of fibrils of these tendons. The ultrasonographic appereance of a normal healthy tendon in the body is composed of multiple, closely settled parallel echogenic lines on longitudinal scanning, whereas multiple echogenic dots or lines are seen in the transverse plane. Ultrasonographic and histological findings of common extensor tendinosis are also similar to findings of degeneration of other tendons in response to chronic overuse such as supraspinatus and patellar tendons ${ }^{[4,6-8]}$. Focal hypoechoic areas correspond histologically to areas of collagen degeneration and intrasubstance fiber rupture. Diffuse heterogenicity defined as lack of echotexture and fibrillar pattern was believed to represent diffuse tendinopathy ${ }^{[4]}$. Partial or even complete tears may occur, if focal hypoechoic areas enlarge and extend to the surface in a normal tendon or in one with underlying diffuse tendinopathy ${ }^{[6]}$. 
Our results showed that heterogenicity and focal hypoechoic area in common extensor tendon were the most common findings in elbows diagnosed with lateral epicondylitis $(40 \%, 35.5 \%$, respectively). In a similar study investingating ultrasonography findings of lateral epicondylitis, Cornell et al. reported that the most common ultrasonographic appearance of lateral epicondylitis was focal hypoechoic area in common extensor tendon on either a normal background or one characterized by a diffuse decrease in echotexture with loss of the normal fibrillar pattern which may correspond to our term "heterogenicity" ${ }^{[4]}$.

In this study, focal areas of calcification in common extensor tendon were not common (13.3\%), consistent with formerly reported data as $5.5 \%-25 \%{ }^{[4,9]}$. Increase in thickness was also a relatively uncommon finding and decrease in tendon thickness was not observed in patients, therefore most patients had normal tendon size. Partial and complete tears were very rare.

Entesophyte was a relatively common finding in patients with lateral epicondylitis, while it was the most common finding in asyptomatic elbows. This may be explained by the degeneration of distal end of the tendon due to frictions against bony epicondyle which becomes apparent before the emergence of the sypmtoms. Although heterogenicity and focal hypoechoic area were lesser when compared to sypmtomatic side, they were other common findings in asymptomatic elbows which may also suggest that the existence of sonographic changes can precede clinical symptoms. According to these results, we think that pathological and ultrasonography findings may appear before the onset of clinical symptoms like Levin et al. hypothesized in their study ${ }^{[1]}$. Furthermore, ultrasonography may be used as a screening test in asymptomatic elbows of patients with lateral epicondylitis in guiding preventive measures to delay future development of contralateral lateral epicondylitis.

Studies demonstrating pathological changes in common extensor tendon, ulnar collateral ligament or heel tendon have been reported ${ }^{[10-14]}$. However, there were limited studies investigating the role of ultrasonography in lateral epicondylitis ${ }^{[1,4,10,12]}$. To our knowledge, this is the first study investigating the correlation of ultrasonographic findings with duration of symptoms and severity of pain. Regarding the duration of symptoms, heterogenicity and focal hypoechoic area were the most seen ultrasonographic findings of early stage disease in this study. This was consistent with the generation of the tendinosis process, since focal hypoechoic areas histologically suits to regions of collagen degeneration and microruptures in early stages of tendinosis ${ }^{[4]}$. Heterogenicity was more common than focal hypoechoic area in late stages of the disease and this may be explained by diffuse background of tendinosis consists of enlarging and extending focal hypoechoic areas. Focal areas of calcification, partial tear and increase in tendon thickness were more common in late stage disease, besides complete tear and peritendinous fluid collection were only seen in late stage disease. However, there was no statistically significant correlation between ultrasonographic findings and the duration of symptoms $(p>.05)$.

Levin et al. reported that symptoms of lateral epicondylitis including pain and tenderness at the region of lateral epicondyle were significantly related with intratendinous calcification, tendon thickening, adjacent bone irregularity, focal hypoechoic regions, and diffuse heterogenecity at ultrasonography ${ }^{[1]}$. We used a scale (VAS) which may subjectively quantify the severity of pain that the patients feel during daily activities including elbow movements. However, we found no statistically significant correlation between severity of pain and ultrasonographic findings.

Therefore, our study demonstrated that there is no relation with clinical sypmtoms and ultrasonographic findings of lateral epicondilitis. This may show the importance of ultrasonographic examination for evaluating the extent of the disease, regardless of severity of symptoms. Patients with early stage disease or low pain scores may have late stage tendinosis findings like calcification or tears. Also some patients with late stage disease or high pain scores may only have early stage tendinosis findings in ultrasonography like focal hypoechoic area or heterogenicity. Therefore, demonstration of ultrasonographic findings may prevent more aggressive therapies. Our patients with partial or complete tears had conservative treatment since their pain scores were low compared with ultrasonographic findings and they responded well 
to conservative treatment in clinical follow-ups. Furthermore, we disagree with the belief that the diagnosis of partial or complete tear should encourage the clinician to refer the patient directly to surgery ${ }^{[4]}$.

We aimed to demonstrate that ultrasonography is a practical method in the demonstration of lateral epicondylitis and could be more commonly used in daily practice. Our results suggest that high resolution ultrasonography can demonstrate the morphology, internal architecture and echogenicity of common extensor tendon in patients with lateral epicondylitis. However, the sonographic findings do not significantly correlate with duration of symptoms and severity of pain. In daily practice, ultrasonography could be used to highlight the extent of the disease without considering the clinical stage or severity of the disease.

Our study has some limitations. First is the relatively small number of patients included in the study. Second is the reference standard was the clinical diagnosis since lateral epicondylitis is essentially a clinical diagnosis and our patients did not undergo to surgery for lateral epicondylitis.

In conclusion, ultrasonography can be used as a complementary imaging method in lateral epicondylitis which confirm the clinical diagnosis and show the extent of the disease. Ultrasonographic findings do not significantly correlate with duration of symptoms and severity of pain in lateral epicondylitis.

\section{References}

[1] Levin D, Nazarian LN, Miller TT, et al. Lateral epicondylitis of the elbow: US findings. Radiology. 2005; $237: 230-234$. PMid:16118152 http://dx.doi.org/10.1148/radiol.2371040784

[2] Scher DL, Wolf JM, Owens BD. Lateral epicondylitis. Orthopedics. 2009: 32. http://dx.doi.org/10.1148/rg.301095078

[3] Walz DM, Newman JS, Konin GP, et al. Epicondylitis: pathogenesis, imaging, and treatment. Radiographics. 2010 ; 30 : 167-184. PMid:11222225

[4] Connell D, Burke F, Coombes P. et al. Sonographic examination of lateral epicondylitis. AJR Am J Roentgenol. 2001; 176: 777-782. http://dx.doi.org/10.2214/ajr.176.3.1760777

[5] Kraushaar BS, Nirschl RP. Tendinosis of the elbow (tennis elbow). Clinical features and findings of histological, immunohistochemical, and electron microscopy studies. J Bone Joint Surg Am. 1999; 81: 259-278. PMid:10073590

[6] Hodgson RJ, O'Connor PJ, Grainger AJ. Tendon and ligament imaging. Br J Radiol. 2012; 85: 1157-1172. http://dx.doi.org/10.1259/bjr/34786470

[7] Potter HG, Hannafin JA, Morwessel RM, et al. Lateral epicondylitis: correlation of MR imaging, surgical and histopathological findings. Radiology. 1995; 196: 43-46. PMid:7784585 http://dx.doi.org/10.1148/radiology.196.1.7784585

[8] Bodne D, Quinn SF, Murray WT, et al. Magnetic resonance images of chronic patellar tendinitis. Skeletal Radiol. 1988 ; $17: 24-28$. PMid:3358133 http://dx.doi.org/10.1007/BF00361451

[9] Nirschl RP, Perrone FA. Tennis elbow: the surgical treatment of lateral epicondylitis. J Bone Joint Surg Am. 1979; 61: 832-839. PMid:479229

[10] Miller TT, Shapiro MA, Schultz W, et al. Comparison of sonography and MRI for diagnosing epicondylitis. J Clin Ultrasound. 2002; 30: 193-202. PMid:11981928 http://dx.doi.org/10.1002/jcu.10063

[11] Martin CE, Schweitzer ME. MR imaging of epicondylitis. Skeletal Radiol. 1998; 27: 133-138. http://dx.doi.org/10.1007/s002560050352

[12] Lee MH, Cha JG, Jin W, et al. Utility of sonographic measurement of the common tensor tendon in patients with lateral epicondylitis. AJR Am J Roentgenol. 2011; 196: 1363-1367. http://dx.doi.org/10.2214/AJR.10.5769

[13] Nazarian LN, McShane JM, Ciccotti MG, et al. Dynamic US of the anterior band of the ulnar collateral ligament of the elbow in asymptomatic major league baseball pitchers. Radiology. 2003; 227: 149-154. PMid:12616000

http://dx.doi.org/10.1148/radiol.2271020288

[14] Cornwall MW, McPoil TG. Plantar fasciitis: etiology and treatment. J Orthop Sports Phys Ther. 1999; 29: 756-760. PMid:10612073 http://dx.doi.org/10.2519/jospt.1999.29.12.756 